\title{
Spectrum of Hypertriglyceridemia at the Onset of Type 2 Diabetes in Ouagadougou
}

\author{
Oumar Guira1,2*, Réné Bognounou22, Lassané Zoungrana1,2, Aline Tonde², André Nagalo1,3, \\ Réné Traore ${ }^{2}$, Joseph Youssouf Drabo1,2 \\ ${ }^{1}$ Unité de Formation et de Recherche en Sciences de la Santé, Université Joseph Ki Zerbo, Ouagadougou, Burkina Faso \\ ${ }^{2}$ Service de Médecine Interne, Centre Hospitalier Universitaire Yalgado Ouédraogo, Ouagadougou, Burkina Faso \\ ${ }^{3}$ Laboratoire de Biochimie, Centre Hospitalier Universitaire Yalgado Ouédraogo, Ouagadougou, Burkina Faso \\ Email: *oumgui@yahoo.fr
}

How to cite this paper: Guira, O., Bognounou, R., Zoungrana, L., Tonde, A., Nagalo, A., Traore, R. and Drabo, J.Y. (2020) Spectrum of Hypertriglyceridemia at the Onset of Type 2 Diabetes in Ouagadougou. Open Journal of Internal Medicine, 10, 83-89.

https://doi.org/10.4236/ojim.2020.101008

Received: January 29, 2020

Accepted: March 13, 2020

Published: March 16, 2020

Copyright (c) 2020 by author(s) and Scientific Research Publishing Inc. This work is licensed under the Creative Commons Attribution International License (CC BY 4.0).

http://creativecommons.org/licenses/by/4.0/

\begin{abstract}
Introduction: Hypertriglyceridemia is a frequent dyslipidemia in type $2 \mathrm{di}-$ abetes. We aimed to determine its prevalence, its typology, and its associated factors in newly type 2 diabetes patients in Ouagadougou. Material and Method: This cross-sectional, descriptive and analytic study has been performed from May 2015 to June 2016 in the Internal medicine department in Yalgado Ouédraogo teaching hospital, Ouagadougou. An accidental sample of newly type 2 diabetes outpatients, naïve of antidiabetics therapy was studied. A 12-hour fasting triglyceridemia was performed; hypertriglyceridemia was defined for triglyceridemia $>1.5 \mathrm{~g} / \mathrm{L}(1.7 \mathrm{mmol} / \mathrm{L})$. Data were analyzed with Epi info 7.1.5.0. Proportions and means were compared respectively with Khi2 or Fisher's test and Student's test with a significance of $\mathrm{p}<5 \%$. Results: One hundred and three patients, i.e. 35 (34\%) men and 68 (66\%) women were included. The sex ratio was 0.51 and the mean age $49.3 \pm 10.1$ years [limits: 24 and 70]. The mean value of triglyceridemia was $1.8 \pm 1.7 \mathrm{mmol} / \mathrm{L}$ [limits: 0.5 and 14.9]. Hypertriglyceridemia was reported in 32 patients (31.1\%): minor $(<4.52 \mathrm{mmol} / \mathrm{L})$ in $26(81.3 \%)$, moderate (between 4.52 and $11.30 \mathrm{mmol} / \mathrm{L})$ in five $(15.6 \%)$ and severe $(>11.30 \mathrm{mmol} / \mathrm{L})$ in one $(3.1 \%)$ patients. Hypertriglyceridemia's phenotype was commonly integrated into a mixed dyslipidemia; it was often combined with a total cholesterolemia increase and a HDL cholesterolemia decrease. In bivariate analysis, hyperglycemia was associated with severe hyperglycemia $(p=0.006)$ and renal failure $(p=0.03)$. Conclusion: Hypertriglyceridemia in type 2 diabetes is frequent and often combined with other lipids disorders. It may need an optimal care.
\end{abstract}

\section{Keywords}

Hypertriglyceridemia, Type 2 Diabetes, Burkina Faso 


\section{Introduction}

Hypertriglyceridemia is a lipid's metabolism disorder reported in 19\% to $70 \%$ type 2 diabetes patients [1], compared, for example, to a global prevalence of 27\% in the Copenhagen General Population Study [2]. Considered for a long time as a cardiovascular risk marker in combination with High-Density Lipoprotein cholesterol (HDL cholesterol) level and Low-Density Lipoprotein cholesterol (LDL cholesterol) particles size decrease, insulin resistance and weight gain, hypertriglyceridemia seem to be according to recent epidemiological data, an independent cardiovascular risk factor [3]. It intervenes especially alongside other factors as a residual cardiovascular risk factor, i.e. the risk of cardiovascular events which persists in subjects despite an achievement of LDL cholesterol level, blood pressure and blood glucose targets [4]. To date, these current evidences are not enough taken into account most of recommendations for cardiovascular risk's evaluation and prevention. In order to describe the spectrum of this lipid's disorder in our diabetes patients, we have performed this study to determine the prevalence, the typology, and the factors associated with hypertriglyceridemia in a cohort of newly type 2 diabetes patients in Ouagadougou.

\section{Patients and Method}

The study was performed from May 2015 to June 2016 in the Internal medicine department, Yalgado Ouédraogo teaching hospital (CHUYO). It was a crosssectional, descriptive and analytic study of an accidental sample of newly type 2 diabetes outpatients. Adults' naïve of antidiabetic therapy (i.e. newly type 2 diabetes patients who have not yet started antidiabetic therapy), who gave their informed verbal consent were included in the study. Pregnant women, patients with another condition of secondary dyslipidemia and patients with hyperglycemic emergency were not included. A 12 hours fasting triglyceridemia was performed within 48 hours following the first consultation and prior to antidiabetic therapy's initiation; it has been performed in the CHUYO's laboratory, using an Architect $\mathrm{Ci} 4000^{\circ}$ automated system. The reagents and controls were the lipid assays provided by the manufacturer (Abbott, USA). Hypertriglyceridemia was defined for triglyceridemia $>1.7 \mathrm{mmol} / \mathrm{L}(1.5 \mathrm{~g} / \mathrm{L})$. Hypertriglyceridemia was minor if $<4.52 \mathrm{mmol} / \mathrm{L}(4 \mathrm{~g} / \mathrm{L})$, moderate if $>4.52 \mathrm{mmol} / \mathrm{L}(4 \mathrm{~g} / \mathrm{L})$ and $<$ $11.30 \mathrm{mmol} / \mathrm{L}(10 \mathrm{~g} / \mathrm{L})$ and severe if $>11.30 \mathrm{mmol} / \mathrm{L}(10 \mathrm{~g} / \mathrm{L})$ [5]. De Gennes classification of dyslipidemia has been used to characterize the phenotype of hypertrigleridemia. Type 2 diabetes was defined according to World Health Organization criteria of diabetes mellitus (two Fasting plasma glucose measurements $\geq 7 \mathrm{mmol} / \mathrm{L}$ or a random plasma glucose $\geq 11 \mathrm{mmol} / \mathrm{L}$ associated with classic diabetes symptoms), an absence of significant ketonuria, and an absence of arguments for secondary diabetes. Data were analyzed with Epi info version 7.1.5.0. Proportions and means were compared respectively with the Khi2 test or the Fisher's test for qualitative data and the Student's test for quantitative data with a significance of $\mathrm{p}<5 \%$. 


\section{Results}

\subsection{Patient's Baseline Data}

One hundred and three patients, i.e. 35 (34\%) men and 68 (66\%) women have been studied. The sex ratio was 0.51 and the mean age $49.3 \pm 10.1$ years [limits: 24 and 70]: $50.0 \pm 9.7$ years for men and $50.4 \pm 10.2$ for women, $\mathrm{p}=0.64$ Smoking was reported in five (4.9\%), alcohol consumption in 20 (19.4\%) and a moderate or intense physical activity practice in $13(12.6 \%)$ patients. Overweight and central obesity concerned respectively $73(69.9 \%)$ and $63(61.2 \%)$ patients. Metabolic syndrome was reported in 39 (37.9\%) patients.

\subsection{Features of Hypertriglyceridemia}

\subsubsection{Prevalence of Hypertriglyceridemia}

The mean value of triglyceridemia in the study's population was $1.8 \pm 1.7 \mathrm{mmol} / \mathrm{L}$ [limits: 0.5 and $14.9 \mathrm{mmol} / \mathrm{L}$ ]. Hypertriglyceridemia was reported in 32 patients (31.1\%), of which 19 (59.4\%) women.

\subsubsection{Severity of Hypertriglyceridemia}

Hypertriglyceridemia was minor in $26(81.3 \%)$ patients, moderate in five $(15.6 \%)$ and severe in one $(3.1 \%)$.

\subsubsection{Phenotypes of Hypertriglyceridemia}

Hypertriglyceridemia was an isolated dyslipidemia in seven patients (21.9\%) and a part of a mixed dyslipidemia in 25 patients (78.1\%); it was often combined with a total cholesterolemia increase and HDL cholesterolemia decrease (31.2\%). The least common phenotype of hypertriglyceridemia combined "Hypertriglyceridemia + Total Hypercholesterolemia + LDL Hypercholesterolemia” (12.5\%). Table 1 shows the distribution of patients according to the phenotypes of hypertriglyceridemia.

\subsubsection{Factors Associated to Hypertriglyceridemia}

The relations between patient's demographic, lifestyle and biological factors and

Table 1. Distribution of newly type 2 diabetes patients according to the phenotype of hypertriglyceridemia $(\mathrm{n}=32)$.

\begin{tabular}{cc}
\hline Phenotype of hypertriglyceridemia & Frequency (\%) \\
\hline Isolated hypertriglyceridemia & $07(21.9)$ \\
Hypertriglyceridemia + Total hypercholesterolemia & $06(18.8)$ \\
Hypertriglyceridemia + Total hypercholesterolemia & $04(12.5)$ \\
+ LDL hypercholesterolemia & \\
Hypertriglyceridemia + Total hypercholesterolemia & $10(31.2)$ \\
HyL hypocholesterolemia & \\
Hypertriglyceridemia + Total hypercholesterolemia & $05(15.6)$ \\
TDL hypercholesterolemia + HDL hypocholesterolemia & $32(100)$
\end{tabular}


hypertriglyceridemia have been determined with a bivariate analysis. Only severe hyperglycemia $(p=0.005)$ and renal failure $(p=0.03)$ was significantly associated to hypertriglyceridemia. Other factors including age, gender, physical activity, alcohol consumption, smoking, obesity or metabolic syndrome were not associated with hyperglycemia in our newly type 2 diabetes patients (Table 2 and Table 3).

Table 2. Relation between demographic factors, lifestyle and hypertriglyceridemia in newly type 2 diabetes patients $(\mathrm{N}=103)$.

\begin{tabular}{|c|c|c|c|}
\hline & \multicolumn{2}{|c|}{ Hypertriglyceridemia } & \multirow[t]{2}{*}{$\mathrm{p}$} \\
\hline & Yes & No & \\
\hline Age (years) & & & 0.87 \\
\hline$<40$ & $08(21.6)$ & $29(78.4)$ & \\
\hline $40-65$ & $23(37.7)$ & $38(62.3)$ & \\
\hline$>65$ & $01(20)$ & $04(80$ & \\
\hline Gender & & & 0.34 \\
\hline Men & $13(37.1)$ & $24(62.9)$ & \\
\hline Female & $19(27.9)$ & $49(62.1)$ & \\
\hline Physical activity & & & 0.19 \\
\hline Yes & $02(15.4)$ & $11(84.6)$ & \\
\hline No & $30(33.3)$ & $60(66.7)$ & \\
\hline Alcohol intake & & & 0.34 \\
\hline Yes & $06(30)$ & $14(70)$ & \\
\hline No & $26(31.3)$ & $57(68.7)$ & \\
\hline Smoking & & & 0.55 \\
\hline Yes & $01(20)$ & $04(80)$ & \\
\hline No & $31(31.6)$ & $67(68.4)$ & \\
\hline
\end{tabular}

Table 3. Relation between clinical factors, biological factors and hypertriglyceridemia in newly type 2 diabetes patients $(\mathrm{N}=103)$.

\begin{tabular}{cccc}
\hline \multicolumn{2}{c}{ Hypertriglyceridemia } & p \\
\hline Yes & No & 0.22 \\
\hline Global obesity & & & \\
Yes & $07(22.6)$ & $24(77.4)$ & 0.85 \\
No & $25(34.7)$ & $47(65.3)$ & \\
Central obesity & & & \\
Yes & $20(31.8)$ & $28(68.2)$ & 0.86 \\
No & $12(30)$ & & \\
Hypertension & $10(32.3)$ & $21(67.7)$ & \\
Yes & &
\end{tabular}




\section{Continued}

\begin{tabular}{cccc}
\hline No & $22(30.6)$ & $50(69.4)$ & \\
Metabolic syndrome & & & 0.12 \\
Yes & $13(41.9)$ & $26(58.1)$ & \\
No & $19(26.4)$ & $5(73.6)$ & 0.005 \\
Glycemia (mmol/L) & $15(22.1)$ & $53(77.9)$ & \\
$<16.5$ & $17(48.6)$ & $18(51.4)$ & 0.03 \\
$>16.5$ & & & \\
Renal failure & $22(35.5)$ & $40(74.5)$ & \\
No & $03(12)$ & $22(88)$ & \\
Minor & $03(60)$ & $02(40)$ & \\
Moderate & &
\end{tabular}

\section{Discussion}

The aim of the study was to determine the prevalence, the typology and the factors associated with hypertriglyceridemia in a cohort of newly type 2 diabetes patients in Ouagadougou. Hypertriglyceridemia was reported in one third patients; it was commonly a part of a mixed dyslipidemia and was associated with severe hyperglycemia and renal failure.

Hypertriglyceridemia was reported in $31.1 \%$ patients. Compared to other studies in newly diabetes patients, this prevalence is similar to $30.0 \%$ reported by Daniel in a native population in Australia [6]. However, it differs from findings of Tian in China, i.e. 69.2\% [7]. The difference could result from genetic, ethnic and environment influences, suggesting why the classic low frequency of hypertriglyceridemia generally reported in black populations [8] [9].

Hypertriglyceridemia was minor in most cases (81.3\%); this corroborates data reporting the usual moderate increase of hypertriglyceridemia in type 2 diabetes. Nevertheless, as in our study, severe hypertriglyceridemia is possible.

According to De Gennes classification of dyslipidemia, hypertriglyceridemia was generally a part of a mixed dyslipidemia (78.1\%). This evidence confers a type IIb or a type III dyslipidemia's phenotype in reference to Fredricksons classification [10] [11] and illustrates the need to be interested in most type 2 diabetes patients suffering from hypertriglyceridemia.

Hypertriglyceridemia was associated with severe hyperglycemia. Qie also in China found such a relation with severe diabetes imbalance [12]. Normally, insulin, by activating lipoprotein lipase (LPL), increases the triglyceride-rich lipoproteins catabolism. Then, an absolute or relative deficiency of insulin in diabetes imbalance explains a proportional accumulation of triglycerides [13] [14]. A similar significant association has been observed with moderate renal failure; this could be explained also by a decrease in triglyceride-rich lipoproteins catabolism, consecutive to the inhibition of LPL by uric acid metabolites [15].

About the methodological procedures, the study had limitations and biases 
which must be taken into account when interpreting our results. Thus, the small number of the study's population reduces the statistical power of the results. Moreover, failure to perform a triglyceridemia control check-up (because of financial limitations) as recommended in dyslipidemia screening could influence the sensitivity of triglyceridemia results provided by the laboratory.

\section{Conclusion}

Hypertriglyceridemia is not a rare dyslipidemia in newly type 2 diabetes patients in Ouagadougou. Its usual combination with other atherogenous lipids disorders could increase its cardiovascular deleterious impact and need an optimal care. Furtherer longitudinal survey has however to be performed to estimate the trends of triglyceridemia in relation to diabetes therapy and its balance.

\section{Conflicts of Interest}

The authors do not declare any conflict of interest in relation to the article.

\section{References}

[1] Tanguy, B. and Aboyans, V. (2014) Dyslipidémie et diabète. Réalités Cardiologiques, 303, 37-41. http://www.realites-cardiologiques.com/

[2] Nordestgaard, B.G. (2016) Triglyceride-Rich Lipoproteins and Atherosclerotic Cardiovascular Disease: New Insights from Epidemiology, Genetics, and Biology. Circulation Research, 118, 547-563. https://doi.org/10.1161/CIRCRESAHA.115.306249

[3] Ziegler, O. Hypertriglycéridémies et risque vasculaire. Hypertriglycéridémies et risque vasculaire. http://Institutdanone.org

[4] Ferrari, R., Aguiar, C., Alegria, E., et al. (2016) Current Practice in Identifying and Treating Cardiovascular Risk, with a Focus on Residual Risk Associated with Atherogenic Dyslipidaemia. European Heart Journal Supplements, 18, C2-C12. https://doi.org/10.1093/eurheartj/suw009

[5] Cugnet-Anceau, C., Moret, M. and Moulin, P. (2011) Existe-il un risque de pancréatite aiguë? Conduite à tenir devant une hypertriglycéridémie. Revue du Praticien, 1110-1116.

[6] Daniel, M., Paquet, C., Kelly, S.J., Zang, G., Rowley, K.G., McDermott, R., et al. (2013) Hypertriglyceridemic Waist and Newly-Diagnosed Diabetes among Remote-Dwelling Indigenous Australians. Annals of Human Biology, 40, 496-504. https://doi.org/10.3109/03014460.2013.806588

[7] Tian, J., Chen, H., Jia, F., Yang, G., Li, S., Li, K., et al. (2015) Trends in the Levels of Serum Lipids and Lipoproteins and the Prevalence of Dyslipidemia in Adults with Newly Diagnosed Type 2 Diabetes in the Southwest Chinese Han Population during 2003-2012. International Journal of Endocrinology, 2015, 1-7. https://doi.org/10.1155/2015/818075

[8] Després, J.P., Couillard, C., Gagnon, J., Bergeron, J., Arthur, S. and James, S. (2000) Race, Visceral Adipose Tissue, Plasma Lipids, and Lipoprotein Lipase Activity in Men and Women. The Health, Risk Factors, Exercise Training, and Genetics (HERITAGE) Family Study. Arteriosclerosis, Thrombosis, and Vascular Biology, 20, 1932-1938. https://doi.org/10.1161/01.ATV.20.8.1932

[9] Morrison, J.A., Barton, B.A., Biro, F.M. and Sprecher, D. (1998) The Conjoint Trait 
of Low High-Density Lipoprotein Cholesterol and High Triglycerides in Adolescent Black and White Males. Metabolism, 47, 514-521. https://doi.org/10.1016/S0026-0495(98)90233-5

[10] Valdigue, P. (2000) Biochimie clinique. 2nd Edition, Toulouse. Edition médicales internationals, $332 \mathrm{p}$.

[11] Boland, B., Chenu, P. and Descamps, O. (2001) Les dyslipidémies en médecine générale: détection, attitude et traitement. Louvain Médical, 119, 79-90.

[12] Qie, L.Y., Sun, J.P., Ning, F., Pang, Z.C., Gao, W.G., Ren, J., et al. (2014) Cardiovascular Risk Profiles in Relation to Newly Diagnosed Type 2 Diabetes Diagnosed by Either Glucose or $\mathrm{HbA}_{1 \mathrm{c}}$ Criteria in Chinese Adults in Qingdao, China. Diabetic Medicine, 31, 920-926. https://doi.org/10.1111/dme.12498

[13] Vergès, B. (2007) Physiopathologie de la dyslipidémie du syndrome métabolique et du diabète de type 2. Nutrition Clinique et Métabolisme, 21, 9-16. https://doi.org/10.1016/j.nupar.2007.01.011

[14] Radermecker, R., Philips, J.-C., Jandrain, B., Paquot, N. and Scheen, A. (2008) L'étude clinique du mois. Contrôle glycémique et morbimortalité cardio-vasculaire chez le patient diabétique de type 2. Résultats des études ACCORD, ADVANCE et VA-Diabetes. Revue médicale de Liège, 63, 511-518.

[15] Beressi, J.-P. Choix d'un antihypertenseur dans l'HTA associée à une dyslipidémie. http://www.edimark.fr/Front/frontpost/getfiles/88.pdf 